\title{
Diversidade estrutural e potencial biológico dos metabólitos secundários de espécies do gênero Myroxylon L.f. (Fabaceae): uma revisão da literatura
}

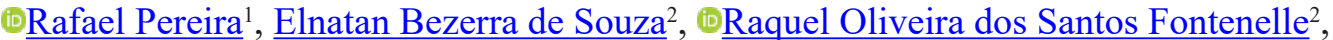

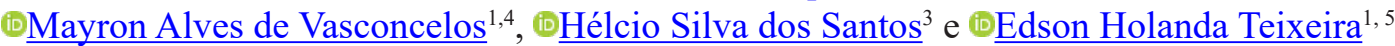

Recebido: 5 setembro 2017; aceito: 8 março 2019

Como citar: Pereira, R., Souza, E.B., Fontenelle, R.O.S., Vasconcelos, M.A., Santos, H.S. \& Teixeira, E.H. 2019. Diversidade estrutural e potencial biológico dos metabólitos secundários de espécies do gênero Myroxylon L.f. (Fabaceae): uma revisão da literatura. Hoehnea 46: e582017. http://dx.doi.org/10.1590/2236-8906-58/2017.

\begin{abstract}
Structural diversity and biological potential of secondary metabolites of species of Myroxylon L.f. (Fabaceae): a review of the literature). Chemical compounds with biological activities isolated from plant and used by the population through extracts and essential oils are promising sources of prospection for new drugs. The genus Myroxylon L. f. showed high pharmacological potential being used in popular medicine for the treatment of various diseases such as wounds, colds, ulcers, headache, and ear infections. This review article presents the main phytochemical and pharmacological characteristics of the species of Myroxylon (M. peruiferum L.f., M. balsamum (L.) Harms and M. pereirae (Royle) Klotzsch), whose studies of biological activities are promising.
\end{abstract}

Keywords: Ethnopharmacology, Papilionoideae, Phytochemistry

RESUMO - (Diversidade estrutural e potencial biológico dos metabolitos secundários de espécies do gênero Myroxylon L.f. (Fabaceae): uma revisão da literatura). Compostos químicos com atividades biológicas isolados de espécies vegetais e usados pela população por meio de extratos e óleo essencial são promissoras fontes de prospecção de novos fármacos. O gênero Myroxylon L. f. apresenta elevado potencial farmacológico, sendo usado na medicina popular para o tratamento de diversas enfermidades, tais como feridas, catarros, úlceras, dores de cabeça e de ouvido. Este artigo de revisão apresenta as principais características fitoquímicas e farmacológicas das espécies do gênero Myroxylon (M. peruiferum L.f., M. balsamum (L.) Harms e $M$. pereirae (Royle) Klotzsch), cujos estudos de atividades biológicas são promissores.

Palavras-chave: Etnofarmacologia, Papilionoideae, Fitoquímica

\section{Introdução}

Os vegetais têm sido utilizados com fins medicinais desde o início da civilização humana para a finalidade de prevenção, tratamento e cura de diversas doenças (De Carvalho et al. 2013). A evolução do conhecimento científico intensificou os estudos sobre plantas medicinais, relacionando a sua composição química com os seus efeitos, confirmando, muitas vezes, a sua utilização popular (Cavalcante et al. 2013). Várias espécies vegetais apresentam constituintes na sua composição química, compostos possuidores de

1. Universidade Federal do Ceará, Departamento de Patologia e Medicina Legal, Rua Monsenhor Furtado, s/n, Rodolfo Teófilo, 60441-750 Fortaleza, CE, Brasil

2. Universidade Estadual Vale do Acaraú, Centro de Ciências Agrárias e Biológicas, Avenida da Universidade, 850, Betânia, 62040-370 Sobral, CE, Brasil

3. Universidade Estadual Vale do Acaraú, Departamento de Química Orgânica, Centro de Ciências Exatas e Tecnologia, Avenida Dr. Guarani, 608, 62042-030 Sobral, CE, Brasil

4. Universidade do Estado do Rio Grande do Norte, Departamento de Ciências Biológicas, Faculdade de Ciências Exatas e Naturais, Rua Prof. Antônio Campos, Costa e Silva, s/n, BR 110, km 48, 59610-090 Mossoró, RN, Brasil

5. Autor para correspondência: edson@ufc.br 
várias atividades biológicas, dentre as quais, podemos destacar as atividades: antimicrobianas, antioxidantes e anticâncer.

A família Fabaceae, também denominada Leguminosae, pertence à ordem Fabales, classe Magnoliopsida e divisão Magnoliophyta (Roskoy et al. 2007). É composta por aproximadamente 19.000 espécies distribuídas em 695 gêneros (Giulietti et al. 2005). Esta família é formada por árvores, arbustos, lianas e ervas e tem distribuição cosmopolita (Di Stasi \& Hiruma-Lima 2002). O Brasil apresenta cerca de 2.827 espécies distribuídas em 222 gêneros, sendo que 1.524 espécies e 16 gêneros são endêmicos (BFG 2015).

É uma das maiores e mais importantes famílias botânicas, devido ao grande número de espécies vegetais utilizadas como fonte de produtos alimentares, medicinais, ornamentais, madeireiros e fornecedores de forragem, fibras, corantes, gomas, resinas e óleos (Di Stasi \& Hiruma-Lima 2002, Watson \& Dallwitz 2009). A família Fabaceae é dividida em três subfamílias: Faboideae, Caesalpinioideae e Mimosoideae (Souza \& Lorenzi 2008).

Dentre os principais gêneros da subfamília Faboideae, destaca-se Myroxylon L. f, cujos estudos fitoquímicos e biológicos de extratos, óleos essenciais e/ou metabólitos secundários estão sendo relatados tanto na medicina cientifica quanto na medicina popular. Apenas duas espécies são encontradas no Brasil: M. peruiferum L. f., amplamente distribuída no país, e M. balsamum, este último apenas é encontrado em território brasileiro no Estado do Acre (Sartori et al. 2015).

Entretanto, a importância de realização de estudos sobre plantas medicinais não reside apenas na caracterização química e física, mas sim na possibilidade de vincular os conteúdos apresentados com determinadas propriedades funcionais bioativas. Nesse contexto, objetivou-se com este artigo apresentar um levantamento bibliográfico do potencial fitoquímico e farmacológico das espécies do gênero Myroxylon: M. peruiferum L. f., M. balsamum (L.) Harms e M. pereirae (Royle) Klotzsch.

\section{Resultados e Discussão}

\section{Família Fabaceae}

Fabaceae constitui uma das famílias botânicas de grande importância econômica e medicinal, destacando-se algumas espécies desta família no tratamento de doenças devido às suas propriedades curativas e terapêuticas. Muitas delas são usadas tanto na farmacologia quanto na medicina popular (Gomes et al. 2008).

Loiola et al. (2010) destacam o uso de plantas da família Fabaceae como recurso medicinal em várias comunidades rurais da Caatinga, mencionando a utilização dessas plantas em rituais religiosos além de enfatizarem a influência desse grupo de plantas na cultura sertaneja.

As espécies desta família são ricas em flavonoides e compostos biossinteticamente relacionados, como o rotenóides e isoflavonoides. Alcaloides, terpenoides e esteroides são exemplos de outras classes de substâncias na qual ocorrem em muitos exemplares da família, já os taninos têm frequência muito baixa se comparada aos flavonoides (Rocha \& Silva et al. 2007). Deste modo, o estudo fitoquímico e farmacológico tem se tornado imprescindível para auxiliar na identificação de substâncias bioativas, comprovando a sua eficácia e tornando o seu uso seguro.

\section{Gênero Myroxylon}

O gênero Myroxylon foi descrito pelo cientista Linnaeus Filius (1781), com base nas amostras provenientes da América do Sul, coletadas por Mutis. As espécies desse gênero Myroxylon são denominadas, popularmente, por diferentes nomes nas áreas de ocorrências, como Chuchupate (Linares \& Bye 1987), Guatemala (Léon \& Alain 1951), Bálsamo, Cabreúvavermelha, Pau-de-incenso, Caboreiba-vermelha, Cabreúva e Pau-vermelho (Lorenzi 1992).

O bálsamo terapêutico mais utilizado é do Peru e do Tolu, obtidos das espécies $M$. pereirae e M. balsamum, respectivamente. Algumas diferenças são abordadas entre as espécies produtora do bálsamo, tanto nos aspectos morfológicos quando no seu aroma. É descrito que essas espécies do gênero Myroxylon têm uso na medicina popular deste o início da civilização, sendo usada contra acarinos e nos tratamentos de feridas e vias respiratórias (Inenami et al. 1984).

De acordo com Fluckiger \& Hanbury (1874), é no caule desse gênero que são encontrados os principais constituístes que são utilizados como ingredientes para a fabricação de pomadas cicatrizantes, aromatizante de xarope e de sabão. Linares \& Bye Jr. (1987), acrescentam ainda, a utilização como medicamento 
para congestão nasal, dores estomacais e reumáticas, e para fabricação de perfumes. Entretanto, trabalhos relacionados a atividades farmacológicas do gênero Myroxylon são escassos na literatura.

\section{Myroxylon peruiferum $\mathrm{L}$. $\mathrm{f}$}

Descrição geográfica - É uma planta de origem nativa, não endêmica no Brasil, com distribuição geográfica confirmadas no Nordeste (Bahia, Ceará, Paraíba), Centro-oeste (Distrito Federal, Goiás, Mato Grosso do Sul, Mato Grosso), Sudeste (Espírito Santo, Minas Gerais, Rio de Janeiro, São Paulo) e Sul (Paraná). Pode ser encontrada no Cerrado e na Mata Atlântica (Sartori 2015).

Conhecimento popular - Myroxylon peruiferum (Fabaceae) é conhecida popularmente como cabriúva, pau-de-bálsamo e bálsamo. É uma planta decídua, heliófita, possuindo cerca de 10 a 20 metros de altura. Suas folhas são compostas pinadas, de ocorrência em quase toda a extensão do país e a madeira é adequada para mobiliário, revestimentos, construção civil, dentre outras atribuições, por causa da sua alta resistência ao apodrecimento (Lorenzi 1998).

Em relação ao conhecimento popular, Lorenzi \& Matos (2002) relatam que a utilização das folhas, frutos e resina do M. peruiferum é ideal para combater feridas e úlceras. Lorenzi \& Matos (2008) acrescentam ainda, a recomendação do uso para asma, reumatismo, catarro, feridas externas, dor de cabeça, torcicolo e tuberculose. Sandoval et al. (1996) mostraram a utilização do extrato aquoso do fruto para aliviar a dor de ouvido. De acordo com Nogueira (1977), todas as partes da planta são consideradas medicinais (a casca, a entrecasca, a madeira, as folhas e o óleo).

Composição química e Propriedades farmacológicas $\mathrm{O}$ estudo da composição química fixa dos extratos benzênico e etanólico do caule de $M$. peruiferum possibilitou o isolamento de isoflavonoides (1-38) (Oliveira et al. 1978, Maranduba et al. 1979). Além de outras classes de metabólicos como a cumarina 2h-1-benzo-piran-2-ona (39) (figura 1) endógena nas sementes de M. peruiferum, a qual está provavelmente relacionada com o efeito alelopático da espécie (Inenami 1984).

O estudo realizado por Gonçalves et al. (2005 e 2007), mostraram que o extrato hidroalcoólico da casca de $M$. peruiferum apresenta atividade antimicrobiana pelo método de difusão em ágar contra isolados clínicos das espécies: Escherichia coli, Enterobacter aerogenes, Klebsiella pneumoniae, Providencia spp, Proteus mrabilis, Pseudomanas aeruginosa, Staphylococus aureus e Salmonella Typhimurium, e sensíbilidade para Streptococcus pyogenes, Shigella flexneri, Shigella sonnei, Staphylococcus epidermidis e Staphylococcus spp.

Matos Neto (2013) demonstrou que o extrato etanolico da casca de $M$. peruiferum apresenta atividade antimicrobiana sobre cepas isoladas a partir de contaminação em alimentos, dentre estas, Staphylococcus aureus (ATCC 25923), Enterococcus faecalis (ATCC 29212), Klebsiella pneumoniae, Aeromonas caviae, Pseudomonas aeruginosa, Vibrio parahaemolyticus, e Escherichia coli. A cabreuvina é o composto mais importante do extrato acetato de etila de $M$. peruiferum, com atividade antibiótica comprovada contra Helicobacter pylori. (Ohsaki et al.1999).

A ação de compostos bioativos sobre fatores de virulência de determinados microrganismos é uma das alternativas mais promissoras para combater microrganismos patogênicos. A partir do extrato aquoso das folhas de $M$. peruiferum, Trentin et al. (2011), constataram a atividade antimicrobiana deste vegetal na formação do biofilme de Staphylococcus epidermidis ATCC 35984.

Muñoz et al. (2000) mostraram que o extrato etanolico do fruto apresentou inibição de $100 \mu \mathrm{g} / \mathrm{mL}$ em atividade antimalárica in vitro, cerca de $44 \%$ de inibição. Relataram ainda, a atividade in vivo em ratos com dose de $1000 \mathrm{mg} / \mathrm{kg}$, com 35\% de inibição.

O óleo essencial da casca de $M$. peruiferum apresentou inibição frente à Leishmania amazonensis de $\mathrm{IC}_{50} 162,25 \mu \mathrm{g} / \mathrm{mL}$. Já para a citotoxicidade contra células monocamada de L6, o óleo essencial apresentou concentração capaz de paralisar 50\% $\left(\mathrm{CC}_{50}\right)$ das células após $24 \mathrm{~h}$ de tratamento de 160 , 80, apresentando baixa toxicidade. (Andrade et al. 2016). Para atividade antioxidante, Silva-Júnior et al. (2015) demonstraram claramente pela primeira vez que os extrato metanólico e aquoso das folhas de $M$. peruiferum apresentam forte atividade antioxidante nos ensaios in vitro realizados.

Recentemente, Pereira et al. (2018) extraíram o óleo essencial de folhas de $M$. peruiferum e isolaram o composto hidróxi- 4',6-dimetóxi-isoflavona a partir do caule da mesma planta. A análise da composição química do óleo essencial mostrou como constituintes majoritários os compostos $\alpha$-pineno (14,85\%), Germacreno D $(17,15 \%)$ e E-cariofileno $(10,78$ 
<smiles>[R10]Oc1ccc2c(=O)c(-c3ccc(OC)c([R6])c3O[R2])coc2c1</smiles>

$1 R_{1}=R_{3}=M e R_{2}=H$

$2 R_{1}=R_{2}=M e R_{3}=H$

$3 R_{1}=R_{2}=R_{3}=M e$

$4 R_{1}=R_{2}=B z R_{2}=M e$

$5 R_{1}=R_{3}=H R_{2}=M e$

$6 R_{1}=R_{3}=M e R_{2}=A c$<smiles>[R2]Oc1c([R1])cc2c(=O)c(-c3ccc(OC)c([R4])c3)coc2c1[R]</smiles>

$19 R_{1}=R_{3}=H R_{2}=M e R_{4}=O M e$

$20 R_{1}=O M e R_{2}=R_{3}==R_{4}=H$

$21 R_{1}=R_{2}=R_{3}=R_{4}=H$

$22 R_{1}=R_{2}=R_{3}=H R_{4}=O H$

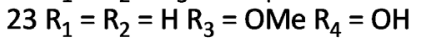

$24 R_{1}=R_{3}=R_{4}=H R_{2}=M e$

$25 R_{1}=R_{3}=R_{4}=H R_{2}=A C$

$26 R_{1}=R_{3}=H R_{2}=A C R_{4}=O A C$<smiles>[R20]Oc1ccc2c(c1)OCC(c1ccc(OC)c([R16])c1O[R2])C2=O</smiles>

$$
\begin{aligned}
& 7 R_{1}=R_{3}=M e R_{2}=H \\
& 8 R_{1}=R_{2}=M e R_{3}=H \\
& 9 R_{1}=R_{2}=R_{3}=M e \\
& 10 R_{1}=R_{2}=B z R_{2}=M e \\
& 11 R_{1}=R_{3}=H R_{2}=M e \\
& 12 R_{1}=R_{3}=M e R_{2}=A C
\end{aligned}
$$<smiles>COc1ccc2c(c1)OC1c3ccc(O)cc3OCC21</smiles>

27<smiles>[R5]c1cc2cc(-c3cc([R3])c([R20])c([R6])c3[R20])oc2cc1[R6]</smiles>

$35 R_{1}=R_{2}=R_{3}=R_{4}=H R_{5}=R_{6}=O M e$

$36 R_{1}=R_{3}=H R_{2}=R_{4}=M e R_{5}=R_{6}=O C_{2} O$

$36 R_{1}=R_{2}=R_{3}=R_{5}=H R_{4}=M e R_{6}=O M e$

$37 R_{1}=R_{5}=H R_{2}=R_{4}=M e R_{3}=R_{6}=O H$

$37 R_{1}=R_{2}=O C H_{2} R_{3}=O M e R_{4}=R_{5}=R_{6}=H$<smiles>[R20]Oc1ccc2c(c1)OCC(c1ccc(OC)c([R6])c1OCC)C2</smiles>

$13 R_{1}=R_{3}=M e R_{2}=H$

$14 R_{1}=R_{2}=M e R_{3}=H$

$15 R_{1}=R_{2}=R_{3}=M e$

$16 R_{1}=R_{2}=B z R_{2}=M e$

$17 R_{1}=R_{3}=H R_{2}=M e$

$18 R_{1}=R_{3}=M e R_{2}=A C$

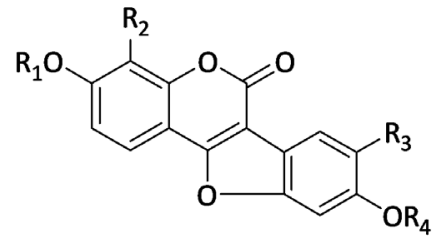

$$
\begin{aligned}
& 28 R_{1}=R_{2}=R_{3}=H R_{4}=M e \\
& 29 R_{1}=R_{2}=H R_{3}=O M e R_{4}=M e \\
& 30 R_{1}=R_{2}=H R_{3}=R_{4}=O C H_{2} \\
& 31 R_{1}=M e R_{2}=O H R_{3}=R_{4}=H
\end{aligned}
$$<smiles>O=C1CC(c2ccc(O)cc2)Oc2cc(O)ccc21</smiles>

38
$33 \mathrm{R}_{1}=\mathrm{Me} \mathrm{R}=\mathrm{H}$

$34 R_{1}=M e R_{2}=O M e$

$35 R_{1}=A c R_{2}=H$

$35 R_{1}=A C R_{2}=O A C$<smiles>O=c1ccc2ccccc2o1</smiles>

39

Figura 1. Representação estrutural das substâncias isoladas de M. peruiferum.

Figure 1. Structural representation of substances isolated from M. peruiferum. 
\%). A atividade antibacteriana e antifúngica do óleo essencial e da isoflavona foram avaliadas sobre espécies relacionadas a infecções. Os resultados obtidos demonstraram que o óleo essencial e a isoflavona apresentaram atividade bacteriostática e/ou bactericida sobre Staphylococcus aureus e Staphylococcus epidermidis; e atividade fungistática e fungicida sobre diferentes estirpes do fungo dermatófito Trichophyton rubrum. Além disso, tanto o óleo essencial como a isoflavona apresentaram significante atividade antioxidante.

\section{Myroxylon balsamum (L.) Harms}

Distribuição geográfica - É uma planta de origem nativa, não endêmica no Brasil, com distribuição geográfica confirmada apenas no Acre (Sartori 2015). Bálsamo do Tolu é obtido a partir M. balsamum uma árvore da família Fabaceae, nativa da Colômbia e Venezuela. Ele também cresce nas Antilhas, principalmente em Cuba. O bálsamo é produzido fazendo incisões na casca das árvores, sob a forma de canais (Bährle-Rapp 2007). Este bálsamo foi incluído na Farmacopeia Britânica, em 1820, com indicação para o tratamento de bronquite, laringite, diarreia, e leucorreia, e como um aromatizante alimentar.

Conhecimento popular - É amplamente utilizado como uma loção para o tratamento de feridas, úlceras e sarna, condicionadores capilares, produtos anticaspa, desodorantes, sabões, cremes, loções, pulverizações e comprimidos para resfriado comum. (Taylor, 1998, BBaylor, 19, 2007, Heinrich et al. 2008).

Composição química e Propriedades farmacológicas O estudo da composição química fixa do extrato etanólico das folhas de $M$. balsamum possibilitou o isolamento de triterpenos (40-53) (Wahlberg et al. 1971, Mathias 2000). Adicionalmente foi constatado também a presença na fração hexânica do isoflavonóide cabreuvina (1) (figura 1) isolado anteriormente de $M$. peruiferum e marcador químico da espécie, além do sesquiterpeno $E$-nerolidol (54) (figura 2) (Simas et al. 2004).

O extrato etanólico assim com as frações hexânica e clorofórmio da casca de M. balsamum apresentaram atividades sobre bactérias resistentes, especialmente, Staphylococcus aureus resistente à meticilina e Pseudomonas aeruginosa isoladas de infecções hospitalares, revelando a sua possível aplicação clínica. O bioensaio com o extrato levou à identificação da chalcona isoliquiritigenina (55) (figura 2), um composto antimicrobiano, apresentando atividade sobre $S$. aureus, $S$. epidermidis e $S$. haemolyticus (Machado et al. 2005).

Estudos realizados por Jeong-Hyun \& Lee (2010) demostraram o potencial antifúngico da resina de $M$. balsamum frente à Malassezia furfur. Enquanto Sales et al. (2016), mostraram em ensaios in vitro, que a tintura mãe de $M$. balsamum apresentou zonas de inibição de crescimento de 12 e $11 \mathrm{~mm}$ de diâmetro para Chalara paradoxa e Fusarium guttiforme respectivamente. Os ensaios foram realizados pelo método de difusão em ágar. As tinturas maternas apresentaram alta difusão no meio de crescimento devido ao seu caráter hidrofílico (Talibi et al. 2012), tornando os grupos químicos ativos disponíveis para os fungos analisados, o que representa uma característica importante na avaliação de novos compostos.

Já Simas et al. (2004), utilizaram a fração hexânica do extrato etanólico da casca de $M$. balsamum e foi caracterizada uma substância ativa, denominada de sesquiterpeno $E$-nerolidol (49) (figura 2), mostrando-se atividade larvicida sobre o A. aegypti, com uma $\mathrm{CL}_{50}$ de $17 \mathrm{ppm}$. Em trabalho anterior, Chantraine et al. (2002) relataram atividade larvicida para a mesma fração, apresentando uma $\mathrm{CL}_{50}$ de 9,0 ppm.

Jankowsky (2005) em seus experimentos sobre atividade anticâncer descreve que o extrato diclorometano e etanolico da casca de M. balsamum apresentaram atividade citostática e citocida para as linhagens celulares: melanoma, carcinoma mamário, carcinoma mamário resistente e carcinoma de grande célula, leucemia, adenocarcinoma ovariano, prostático, colorretal e de células renais.

No que se refere à toxicidade, Popova et al. (2001) demostraram que os extratos com éter de petróleo, etanol e n-butanol de $M$. balsamum apresentaram toxicidade sobre Artemia salina com $\mathrm{IC}_{50} 1,8,1,7,0,4$, 0,4 e 23, respectivamente.

Em relação à atividade antioxidade, estudos realizados por Saleh et al. (2010) mostraram que o óleo essencial de M. balsamum apresentou mais de $90 \%$ de inibição do radical livre 2,2-difenil-1-picril-hidrazil (DPPH) nas concentrações de $100 \mathrm{mg} / \mathrm{mL}, 25 \mathrm{mg} / \mathrm{L}$ e $5 \mathrm{mg} / \mathrm{mL}$. Produtos naturais estão em crescente demanda para a fabricação de alimentos, cosméticos e produtos farmacêuticos. O balsamo do Tulo é um agente bloqueador solar bem conhecido na qual apresenta um fator de proteção solar (FPS) adequado, quando comparado com o ácido aminobenzóico (ElShaer 2006). 


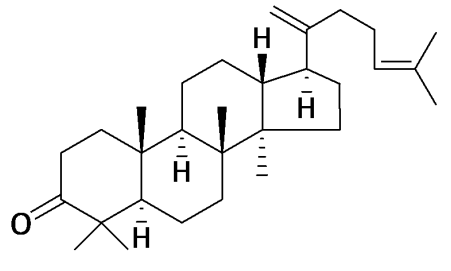

40

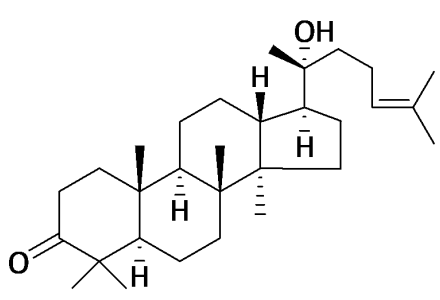

41

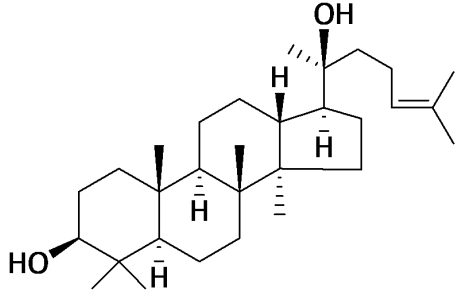

42

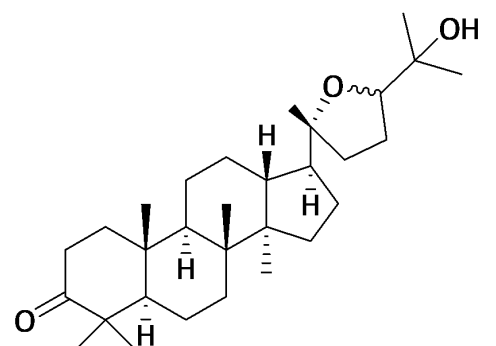

43

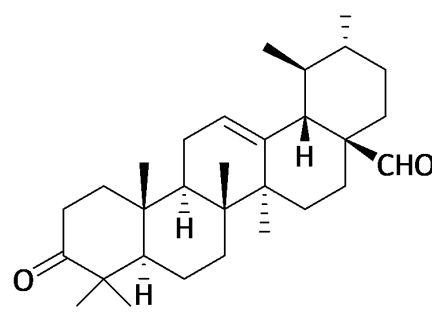

44

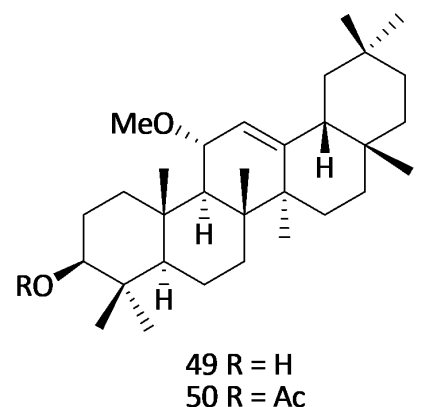

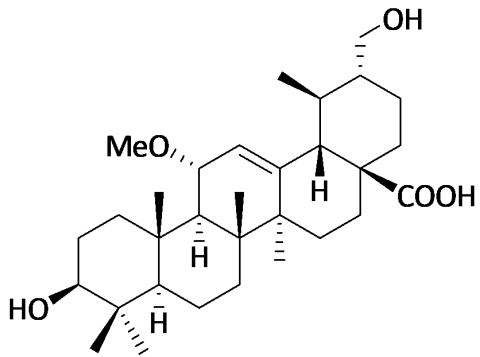

53

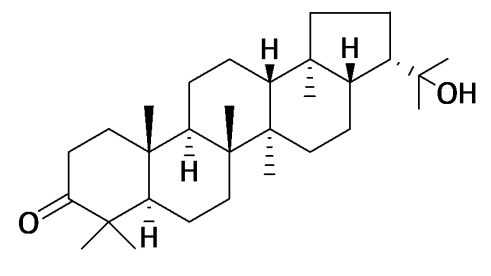

45

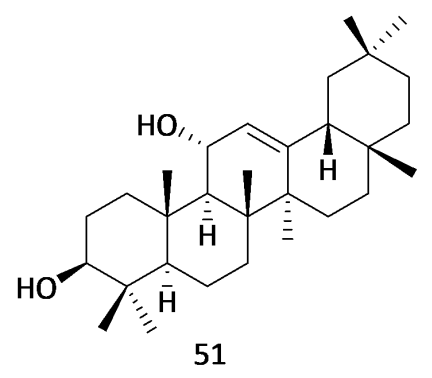

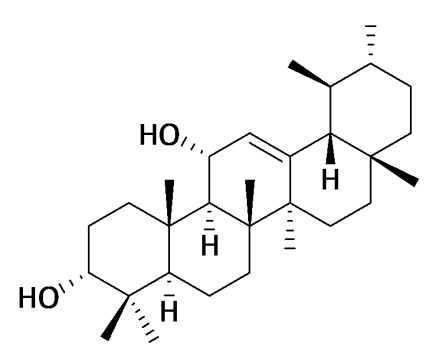

52<smiles>O=C(/C=C/c1ccc(O)cc1)c1ccc(O)cc1O</smiles>

55

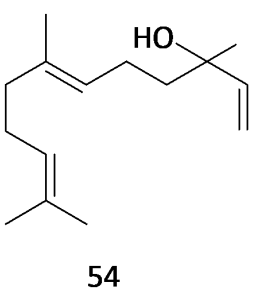


<smiles>OCc1ccccc1</smiles>

56

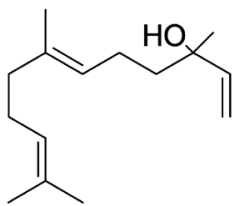

60<smiles>O=C(O)c1ccccc1</smiles>

57<smiles>COc1cc(C=O)ccc1O</smiles>

58<smiles>O=C(O)/C=C/c1ccccc1</smiles>

59<smiles>O=C(/C=C/c1ccccc1)OCc1ccccc1</smiles>

61<smiles>O=C(OCc1ccccc1)c1ccccc1</smiles>

62

Figura 3. Representação estrutural das substâncias identificados no óleo essencial de M. pereirae.

Figure 3. Structural representation of the substances identified in the essential oil of M. pereirae.

\section{Myroxylon pereirae (Royle) Klotzsch}

Distribuição geográfica - M. pereirae é uma espécie nativa da América Central (Robbers et al. 1996).

Conhecimento popular - Tradicionalmente é utilizada no tratamento contra câncer (Hartwell 1970). Esta espécie produz um bálsamo denominado como bálsamo do Peru, assim chamado porque um dos primeiros lugares que foi vendido foi no Porto no Peru (Robbers et al. 1996). As folhas e caule foram usados por tribos indígenas do México e América Central para o tratamento de asma, catarro, reumatismo e feridas externas (Taylor 1998).

Composição química e Propriedades farmacológicasA análise do óleo essencial da resina de $M$. pereirae possibilitou a identificação dos constituintes álcool benzílico (56), ácido benzóico (57), vanilina (58), ácido cinâmico (59), (E)-nerolidol (60), benzoato de benzila (61) e cinamato de benzila (62) (figura 3) (Seo et al. 2012).

M. pereirae possui propriedades antissépticas, antibacteriana e antiparasitária (especialmente para a sarna) (Blumenthal \& Klein 1995). O Bálsamo do Peru tem sido vastamente estudado como um agente alergênico em muitas áreas, e as suas substâncias são encontradas em muitos produtos utilizados na sociedade, tais como alimentos e produtos de higiene, como sabonetes, desodorantes, perfumes e outros. Farnesol, uma substância que está presente no bálsamo do Peru, mostrou uma elevada percentagem das alergias de contato (Schnuch et al. 2004).
De fato, M. pereirae pode causar dermatite de contato alérgica, contato urticária, reação fototóxica (Kroon 1983), reações de contato não imunológico (Tanaka et al. 2004, Katsarou et al. 1999), assim como a susceptibilidade a uma reação imediata. Atualmente essa espécie é considerada um marcador de alergia em perfumes (Hjorth1961, Larsen 1985), sendo que a presença de muitos alérgenos em perfumes ocorre pela possível presença de componentes presentes no bálsamo do Peru ou estão intimamente relacionados a eles (Avalos-Peralta et al. 2005).

M. pereirae é uma planta amplamente utilizada em preparações tópicas para o tratamento de feridas, cicatrização de enxerto de pele, úlceras indolentes, sarna, assaduras, hemorroidas, prurido anal, escaras, intertrigo, eczema e outros; em tônico capilar e preparações anticaspa, sprays de higiene feminina, e como um fixador ou a fragrância (Opdyke 1974, Carson et al.2003).

O óleo essencial e os seus constituintes apresentaram atividade larvicida contra $A$. aegypti, apresentando grandes vantagens, tais como um efeito mínimo de degradação sobre o ecossistema com pouco resíduo na água. (Seo et al. 2012). Em relação à atividade antioxidante, estudos realizados por Lin et al. (2009) mostraram que o óleo essencial de $M$. pereirae apresentou atividade antioxidante nos testes de inibição do radical livre DPPH.

\section{Conclusão}

Apesar do crescente uso de plantas medicinais na medicina popular, é importante à comprovação 


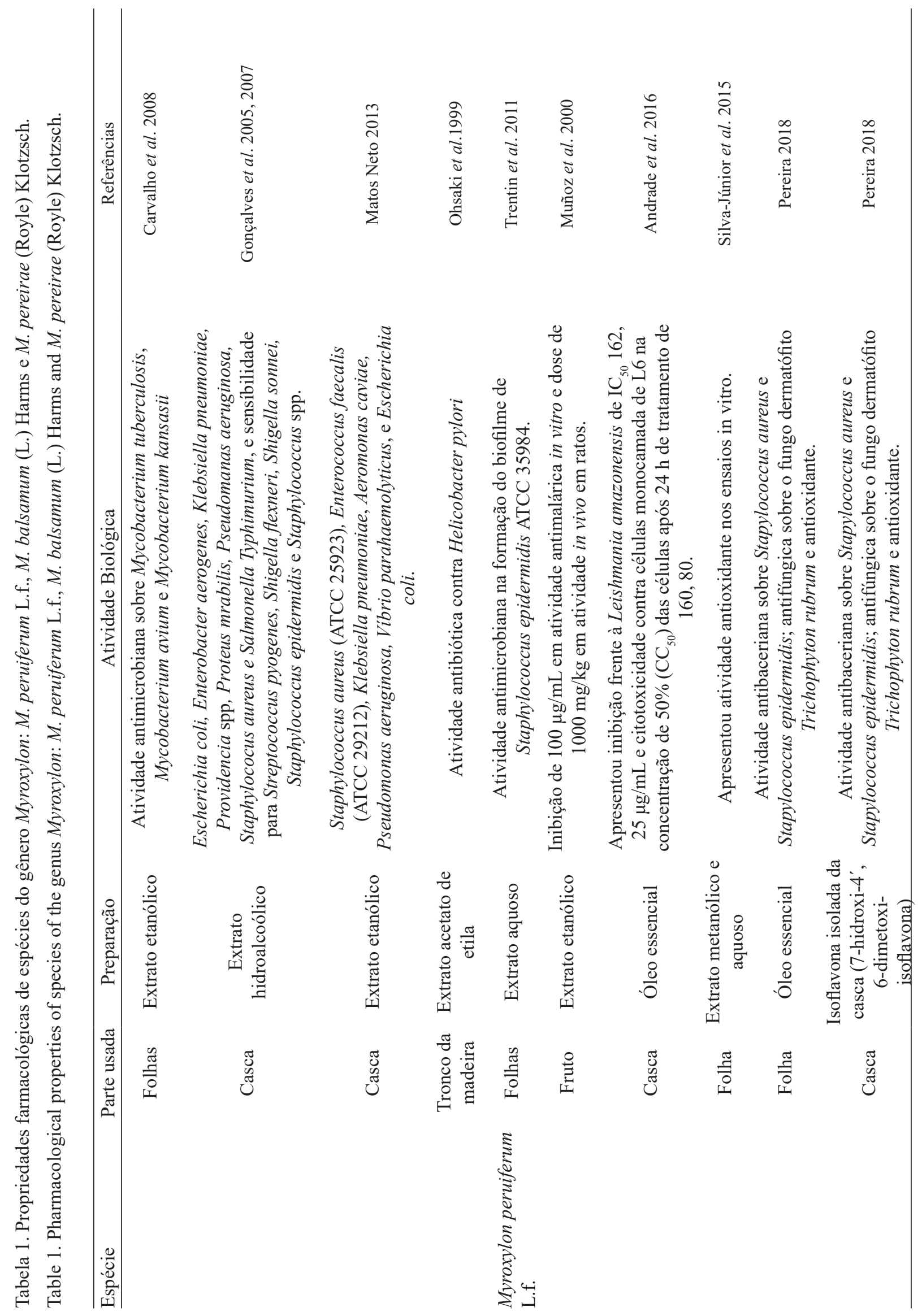


do seu efeito farmacológico. Os resultados desta revisão confirmam o promissor potencial deste gênero como fonte de substâncias bioativas. Contudo, é extremamente necessária uma investigação cientifica mais aprofundada das espécies do gênero Myroxylon partir de seus extratos brutos, frações e óleos essenciais com o objetivo de utilizá-los especificadamente no tratamento de doenças.

\section{Literatura citada}

Andrade, M.A., Azevedo, C.S., Motta, F.N., Santos, M.L., Silva, C.L., Santana J.M., \& Bastos, I.M.D. 2016. Essential oils: in vitro activity against Leishmania amazonensis, cytotoxicity and chemical composition. BMC Complementary and Alternative Medicine 16:1-8.

Avalos-Peralta, P., García-Bravo, B., \& Camacho, F.M. 2005. Sensitivity to Myroxylon pereirae resin (balsam of Peru). A study of 50 cases. Contact Dermatitis 52: 304-306.

Bährle-Rapp, M. 2007. Myroxylon balsamum var. pereirae. In: Springer Lexikon Kosmetik und Körperpflege. Springer Medizin Verlag, Heidelberg.

BFG. 2015. Growing knowledge: an overview of Seed Plant diversity in Brazil. Rodriguésia 66: 1085-1113.

Blumenthal, J.A., Jiang, W., Waugh, R., Frid, D.J., Morris, J.J., Coleman, R.E., Hanson, M., Babyak, M., Thyrum, E.T., Krantz, D.S. \& O'Connor, C.D. 1995. Mental stress induced ischemia in the laboratory and ambulatory ischemia during daily life: Associatio and hemodynamic features. Circulation 92: 2102-2108.

Carson, S.N., Wiggins, C., Overall, K. \& Herbert, J. 2003. Using a Castor Oil-Balsam of Peru-Trypsin Ointment to Assist in Healing Skin Graft Donor Sites. Ostomy Wound Manage. 49: 60-64.

Cavalcante, G.M., Neto, J.F.L., Bomfim, E.O. \& Santos, M.F. 2013. Atividade antimicrobiana de Artocarpus heterophyllus Lam. (Moraceae) sobre o desenvolvimento de Streptococcus pneumoniae e Escherichia coli. Scientia Plena 9:1-7.

Chantraine, J.M., Laurent, D., Ballivian, C., Saavedra, G., Ibañez, R. \& Vilaseca, L.A.1998. Insecticidal activity of essential oils on Aedes aegypti larvae. Phytotherapy Research 12: 350-354.

De Carvalho, R.B.F., Almeida, A.A.C., Freitas, R.M., Lima, L.S., David, J.P., David, J.M. \& Feitosa, C.M. 2013. Composição química e atividade anticolinesterásica de uma fração ativa do extrato de folhas de Citrus limon (L.) Burm. Quimica Nova 36: 1375-1379.

Di Stasi, L.C. \& Hiruma-Lima, C.A. 2002. Plantas medicinais na Amazônia e na Mata Atlântica. 2 ed. revista e ampliada, São Paulo.

El-Shaer, N.S. 2006. Evaluation of some natural products and plant acids against UVB radiation (in vitro). Alexandria journal of pharmaceutical sciences 20: 7-11.
Fluckiger, F. A. \& Hanbury, D. 874. Balsamum tolunatum. Pharmacographia 15: 177-184.

Giulietti, A.M., Harley, R.M., Queiroz, L.P., Wanderley, M.G. \& Berg, C.V.B. 2005. Biodiversidade e conservação das plantas no Brasil. Megadiversidade 1: 52-60.

Giulietti, A.M., Harley, R.M., Queiroz, L.P., Wanderley, M.G. \& Berg, C.V.B. 2005. Biodiversidade e conservação das plantas no Brasil. Megadiversidade 1: $52-60$.

Gomes, E.C.S., Barbosa, J., Vilar, F.C.R., Perez, J.O., Vilar, R.C. Freire, J.L.O., Lima, A.N. \& Dias, T.J. 2008. Plantas da Caatinga de uso terapêutico: Levantamento Etnobotânico. Engenharia Ambiental, Espírito Santo do Pinhal 5: 74-85.

Gonçalves, A.L, Filho, A.A. \& Menezes, H. 2005. Estudo comparativo da atividade antimicrobiana de extratos de algumas árvores nativas. Arquivos do Instituto Biológico 72: 353-358.

Gonçalves, A.L. 2007. Estudo da atividade antimicrobiana de algumas árvores medicinais nativas com potencial de conservação/recuperação de florestas tropicais. Tese de doutorado, Universidade Estadual Paulista Júlio de Mesquita Filho, Rio Claro.

Gottlieb, O.R. \& Magalhaes, M.T. 1959. The chemistry of Brazilian Leguminosae. I. Isolation of 3',4',7-trimethoxyisoflavone (cabreuvin) from Myroxylon balsamum and Myrocarpus fastigiatus. Anais da Associação Brasileira de Química 18: 89-97.

Harborne, J.B., Gottlieb, O.R. \& Magalhaes, M.T. 1963. Occurrence of the isoflavone afromosin in cabreuva wood. The Journal of Organic Chemistry 28: 881-882.

Hartwell, J.L. 1970. Plants used against cancer. A survey. Lloydia 33: 97-194.

Heinrich, M., Barnes, J., Gibbons, S. \& Williamson, E.M. 2008. Fundamentals of Pharmacognosy and Phytotherapy. Churchill Livingstone, Edinburgh.

Hjorth, N. 1961. Eczematous allergy to balsams, allied perfumes and flavouring agents, with special reference to balsam of Peru. Acta Derm Venereol (Stockh) 41: 1-216.

Inenami, T.O. 1984. Estudo do inibidor de germinação em sementes de Myroxylon peruiferum L.f. (Cabriúva)Leguminosae. Tese de Doutorado. Universidade Estadual de Campinas, Campinas.

Jankowsky, L. 2005. Atividade farmacológica de extratos obtidos de a partir de resíduos madeireiros. Tese de Doutorado. Universidade Estadual de Campinas, Campinas.

Katsarou, A., Armenaka, M., Ale, I., Koufou, V. \& Kalogeromitros, D. 1999. Frequency of immediate reactions to the European standard series. Contact Dermatitis 41: 276-279.

Kroon, S. 1983. Standard photopatch testing with Waxtar1, paraaminobenzoic acid, potassium dichromate and balsam of Peru. Contact Dermatitis 9: 5-9. 
Larsen, W.G. 1985. Perfume dermatitis. Journal of The American Academy of Dermatology 12: 1-9.

Lee, J-H \& Lee J-S. 2010. Chemical Composition and Antifungal Activity of Plant Essential Oils against Malassezia furfur. Journal of Microbiology and Biotechnology 38: 315-321.

Leon, H. \& H. Alain. 1951. Flora de Cuba 2. Dicotiledoneas: Casuarinaceas a Meliaceas. Contribuciones Ocasionales del Museo de Historia Natural del Colegio "De La Salle" 10: 1-456.

Lin, C. W., Yu, C.W, Wu, S.C. \& Yih, K. H. 2009. DPPH Free-Radical Scavenging Activity, Total Phenolic Contents and Chemical Composition Analysis of FortyTwo Kinds of Essential Oils. Journal of Food and Drug Analysis 17: 386-395.

Linares, E. \& Bye Jr., R.A. 1987. A study of four medicinal plant complexes of Mexico and adjacente United States. journal of ethnopharmacology. 19: 153-183.

Linnaeus filius, C. 1781. Myroxylon peruiferum. Supplementum Plantarum 34: 232- 234.

Loioloa, M.I.B., Paterno, G.B.C., Diniz, J.A., Calado, J.F. \& Oliveira, A.C. P. 2010. Leguminosas e seu potencial de uso em comunidades rurais de São Miguel do Gostoso-RN. Revista Caatinga, Mossoró 23: 59-70.

Lorenzi, H. \& Matos, F.J.A. 2008. Plantas Medicinais no Brasil: nativas e exóticas. Nova Odessa, Plantarum.

Lorenzi, H. 1992. Árvores brasileiras: manual de identificação e cultivo de plantas arbóreas nativas do Brasil. Ed. Plantarium, Nova Odessa.

Lorenzi, H. 1998. Árvores Brasileiras: manual de identificação e cultivo de plantas arbóreas nativas do Brasil. Plantarium, Nova Odessa.

Lorenzi, H.E. \& Matos, F.J. A. 2002. Plantas medicinais no Brasil/ Nativas e exóticas. Nova Odessa, Instituto Plantarum.

Machado, T.B., Leal, I.C.R., Kuster, R.M., Amaral, A.C.F., Kokis, V., De Silva, M.G. \& Netto Dos Santos, K.R. 2005. Brazilian phytopharmaceuticals-evaluation against hospital bacteria. Phytotherapy Research19: 519-525.

Maranduba, A., Oliveira, A.B., Oliveira, G.G., Reis, J.E.P. \& Gottlieb, O.R. 1979. Isoflavonoids from Myroxylon peruiferum. Phytochemistry 18: 815-817.

Mathias, L., Vieira, I.J.C., Braz-Filho, R. \& Rodrigues Filho, Edson. 2000. ANew Pentacyclic Triterpene Isolated from Myroxylon balsamum (syn. Myroxylon peruiferum). Journal of the Brazilian Chemical Society 11: 195-198.

Matos Neto, F.A. 2013. Abordagem etnobotânica de plantas medicinais usadas para tratamento de lesões de pele associado, ou não, a bactérias ou fungos no município de Itapajé, Ceará, Brasil. Trabalho de conclusão de curso, Universidade Estadual Vale do Acaraú, Sobral.

Muñoz, V., Sauvain, M., Bourdy, G., Arrázola, S., Callapa, J., Ruiz, G., Choque, J. \& Deharo, E. 2000. A search for natural bioactive compounds in Bolivia through a multidisciplinary approach Part III. Evaluation of the antimalarial activity of plants used by Alteños Indians. Journal of Ethnopharmacology 71: 123-131.
Naves, Y.R. 1949. Presence of nerolidol in papilionaceous essential oils. Helvetica Chimica Acta 32: 2181-2185.

Ohsaki, A., Takashima, J., Chiba, N. \& Kawamura, M. 1999.Microanalysis of a selective potent antiHelicobacter pylori compound in a Brazilian medicinal plant, Myroxylon peruiferum and the activity of analogues. Bioorganic \& Medicinal Chemistry Letters 9: 1109-1112.

Oliveira, A.B., Iracema, M., Madruga, L.M. \& Gottlieb, O.R. 1978. Isoflavonoids from Myroxylon balsamum. Phytochemistry 17: 593-595.

Opdyke, D.L.J. 1976. Monographs on fragrance raw materials: fennel oil, bitter. Food and Cosmetics Toxicology 14: 309-310.

Pereira, R. 2018. Atividade antimicrobiana e antioxidante de metabólitos bioativos e óleo essencial de plantas da caatinga: Myroxylon peruiferum L.f e Combretum leprosum. Dissertação de Mestrado, Universidade Federal do Ceará, Fortaleza.

Popova, M., Bankova, Vassya., Chimov, Angel. \& Silva, M.V. 2002. A scientific note on the high toxicity of propolis that comes from Myroxylon balsamum trees. Apidologie 33: 87-88.

Robbers, J.E., Speedie, M.K. \& Tyler,V.E.1996. Pharmacognosy and Pharmacobiotechnology. Williams \& Wilkins, Baltimore.

Rocha e Silva, H., Silva, C.C.M., Caland-Neto, L.B., Lopes, J.A.D., Citó, A. M.G.L. \& Chaves, M.H. 2007. Constituintes químicos das cascas do caule de Cenostigma macrophyllum: ocorrência de colesterol. Química Nova 30: 1877-1881.

Roskoy, Y.R., Bisby, F.A., Zarucchi, J.L., Schrire B.D. \& White R.J. 2007. International Legume Database and Information Service (ILDIS). Disponível em http:// www.ildis.org (acesso em 10-IV-2017).

Sahoo, S., Singh, S. \& Nayak, S. 2014. Chemical composition, antioxidant and antimicrobial activity of essential oil and extract of Alpinia malaccensis Roscoe (Zingiberaceae). International Journal of Pharmacy and Pharmaceutical Sciences 6: 183-188.

Sales, M.D.C., Costa, H.B., Fernandes, P.M.B., Ventura, J.A., \& Meira, D.D. 2016. Antifungal activity of plant extracts with potential to control plant pathogens in pineapple. Asian Pacific Journal of Tropical Biomedicine 6: 26-31.

Sandoval, P., Choque, J. \& Uriona, P. 1996. Cartilla popular sobre las plantas utiles de los Alteños de MizqueCochabamba. Fonama-Cibe-Umsa, Cochabamba, Bolivia.

Sartori, A.L.B., Lewis, G.P., Mansano, V.F. \& Tozzi, A.M.G.A. 2015. A revision of the genus Myroxylon (Leguminosae: Papilionoideae). Kew Bulletin 70: 1-12.

Schnuch, A., Lessmann, H., Geier, J., Frosch, P.J. \& Uter, W. 2004. Contact allergy to fragrances: frequencies of sensitization from 1996 to 2002. Results of the IVDK. Contact Dermatitis 50: 65-76. 
Seo, S-M., Park, H-M. \& Park, II-K. 2012. Larvicidal Activity of Ajowan (Trachyspermum ammi) and Peru Balsam (Myroxylon pereira) Oils and Blends of Their Constituents against Mosquito, Aedes aegypti, Acute Toxicity on Water Flea, Daphnia magna, and Aqueous Residue. Journal of Agricultural and Food Chemistry 60: 5909-5914.

Silva-Júnior, C.A., Silva, L.C.N., Souza, R.M.,Aranda-Souza, M., Pereira, D.S.T., Silva, M.V. \& Correia, M.T.S. 2015. Myroxylum peruiferum: antioxidant activity and phenolic content. Current Topics in Phytochemistry 12: 21-25.

Simas, N.K., Lima, E.C., Conceição, S.R., Kuster, R.M. \& Oliveira Filho, A.M. 2004. Produtos naturais para o controle da transmissão da dengue- Atividade larvicida de Myroxylon balsamum (óleo vermelho) e de terpenóides e fenilpropanóides. Química Nova 27: 46-49.

Souza, V. C. \& Lorenzi, H. 2008. Botânica Sistemática: guia ilustrado para identificação das famílias de fanerógamas nativas e exóticas no Brasil, baseado em APGII. 2 ed. Nova Odessa, SP: Instituto Plantarum.

Talibi, I., Askarne, L., Boubaker, H., Boudyach, E.H., Msanda, F., Saadi, B. \& Aoumar, A. A. B. 2012. Antifungal activity of some Moroccan plants against Geotrichum candidum, causal agent of postharvest citrus sour rot. Crop Protection 35: 41-46.
Tanaka, S., Matsumoto, Y., Dlova, N., Ostlere, L.S., Goldsmith, P.C., Rycroft, R.J.G., Basketter, D.A., White, I.R., Banerjee, P. \& Mcfadden, J.P. 2004. Immediate contact reactions to fragrance mix constituents and Myroxylon pereirae resin. Contact dermatitis 51:20-21.

Taylor, L. 1998. Herbal secrets of the rainforest: the healing power of over 50 medicinal plants you should know about. Rocklin: Prima Healh.

Trentin, D. S., Giordani, R.B., Zimmer, K. R., Silva A. G., Silva, M. V., Correia, M.T. S., Baumvol, I. J. R. \& Macedo, A. J. 2011. Potential of medicinal plants from the Brazilian semi-arid region (Caatinga) against Staphylococcus epidermidis planktonic and biofilm lifestyles. Journal of Ethnopharmacology 137: 327-335.

Wahlberg, I., Hielte, M.B., Karlsson, K. \& Enzell, C.R. 1971. Constituents of commercial Tolu balsam. Acta Chemica Scandinavica 25: 3285-3295.

Watson, L. \& Dallwitz, M.J.2009. The families of flowering plants: descriptions, illustrations, identification, and information retrieval. Disponível em http://delta-intkey. com (acesso em 10-V-2017). 